\title{
Chapter 2 \\ Global Strategies and Response \\ Measures to the Influenza A (H1N1) \\ Pandemic
}

\subsection{The Global Pandemic Influenza A (H1N1)}

As an infectious respiratory disease, influenza is prone to cause pandemics for its fast mutation, easy dissemination, susceptibility to humans, and its elusive nature in terms of treatment. Three influenza pandemics occurred in the 20th century which caused huge losses worldwide. According to historical estimations, in 1918 the Spanish Influenza (H1N1) may have resulted in roughly 20-40 million deaths worldwide. ${ }^{1}$ It was so deadly that some scholars view it as one of the deadliest events in human history. The Asian Influenza (H2N2) in 1958 claimed about two million lives and the Hong Kong Influenza (H3N2) in 1968 caused an estimated one million deaths.

This pandemic originated from a new Influenza A virus that was discovered in North America in March-April of 2009. The spread of this virus sparked the first influenza pandemic of the century, which swept the globe in less than half a year. By June 11th, 2009, 28,744 confirmed cases, including 144 deaths, had been reported in 74 countries and regions in North America, South America, Europe, Oceania, Asia and Africa, and on that very day the WHO raised the alert level to Phase 6 and declared it a global Influenza A pandemic.

Two peak phases occurred during the pandemic on a global and regional level, one in the spring of 2009 and the other in the autumn and winter period of the same year. Beginning in April 2010, the global death rate from the pandemic decelerated along with its scope, as shown in Figs. 2.1 and 2.2.

By August 1st, 2010, more than 214 countries and regions throughout the world reported confirmed cases of Influenza A, with a total of 18,449 deaths. The WHO believed that actual number of cases and deaths exceeded those reported.

On August 10th, 2010, the WHO declared the end of the Influenza A Pandemic, and announced the beginning of the global post-pandemic period. According to

\footnotetext{
${ }^{1}$ WHO (2009a).
}

(C) Social Sciences Academic Press and Springer Nature Singapore Pte Ltd. 2019 


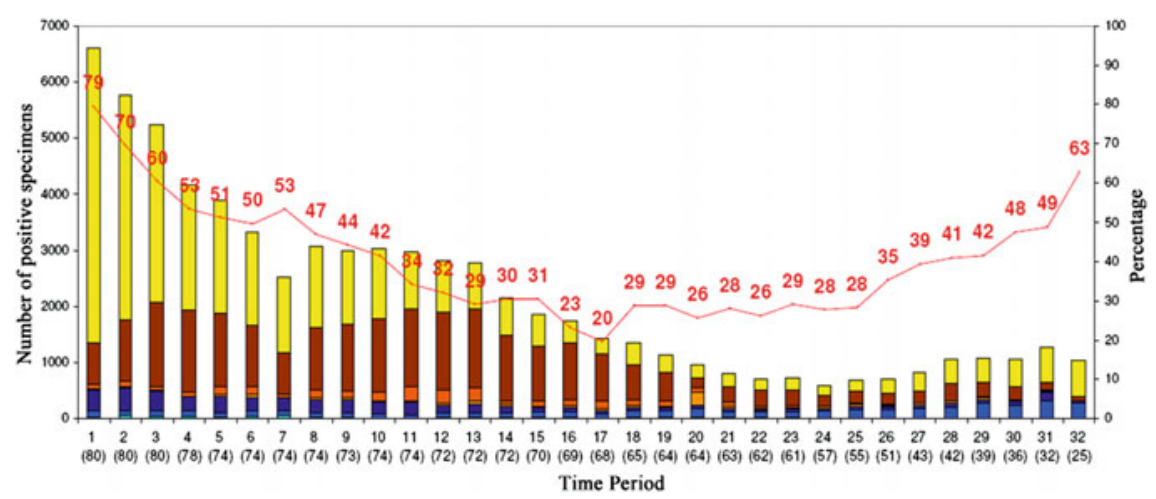

Fig. 2.1 Global Spread of Influenza Viruses from April 19th, 2009 through August 14th, 2010 (WHO. Weekly virological update on 26 August 2010. http://www.who.int/csr/disease/swineflu/ laboratory27_08_2010/en/index.html)

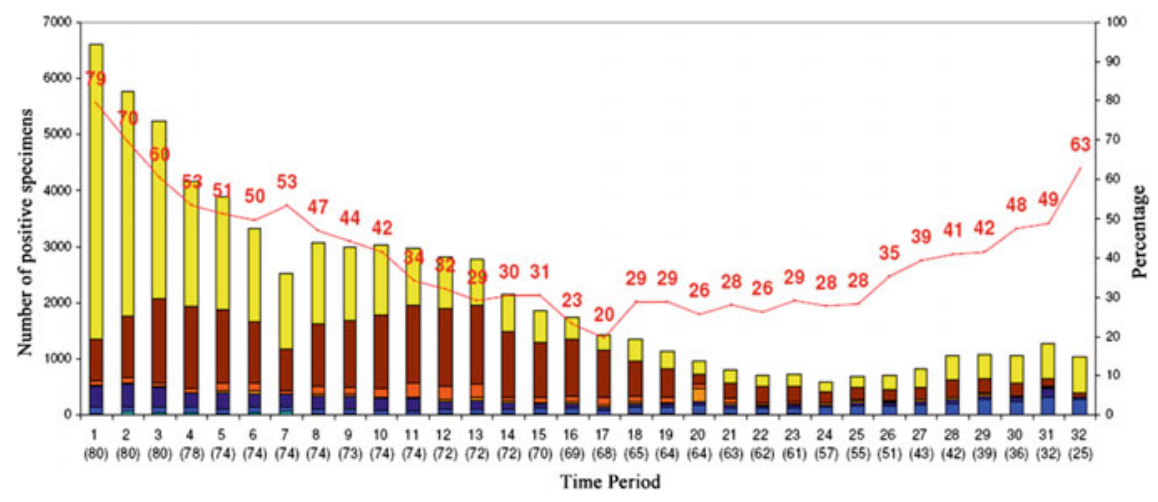

Fig. 2.2 Global Spread of Influenza Viruses from January 3rd, 2010 through August 14th, 2010 (WHO. Weekly virological update on 26 August 2010. http://www.who.int/csr/disease/swineflu/ laboratory27_08_2010/en/index.html)

analysis by the WHO, after the global peak in the winter of 2009 , there were no signs of any further widespread dissemination of the virus, thus proving the end of the Influenza pandemic. Nevertheless, the organization warned that entering the post-pandemic period didn't mean the Influenza A virus would disappear completely, as epidemic outbreaks were still likely to occur in some regions. Additionally possibilities of virus variation were evident and so countries were advised to be on alert during this time. 


\subsection{WHO's Global Pandemic Strategies and Response Measures}

In response to the threat of a global influenza pandemic, the WHO as per the International Health Regulations 2005 (IHR 2005), put a large amount of work into global prevention and control efforts, and also adjusted prevention and control strategy priorities to fall in line with this global influenza outbreak. Countries worldwide have been proactive in their responses to the WHO's strategies and recommendations.

\subsubsection{Capacity Building and Preparedness}

\subsubsection{Publishing the Pandemic Influenza Preparedness and Response}

In order to tackle possible influenza pandemics and minimize losses, in 1999 the WHO published its official guidance, the Influenza Pandemic Plan: the Role of the WHO and Guidelines for National and Regional Planning, which was then later revised in 2005 and 2009, respectively. ${ }^{2}$ In the 2005 revised WHO Global Influenza Preparedness Plan, an influenza pandemic was divided into six different phases: Phases 1-2 are interpandemic, i.e., no new influenza viruses have been detected in humans but an influenza virus subtype is circulating among animals and could potentially pose a threat to humans; Phases 3-5 consist of the pandemic alert phases where a new influenza virus has been detected in humans but its spread among humans remains limited; Phase 6 is the warning phase, declaring that the new influenza virus has spread widely across human populations.

In its 2009 revision of the Pandemic influenza preparedness and response, the WHO retained the use of a six-phase approach, but made some changes to the criteria. Phases 1-3 are characterized by the transmission of an influenza virus among animals and few humans, and correlate with preparedness, including capacity building and response planning activities. Phase 4 is characterized by sustained human-to-human transmission of an influenza virus, while in Phases 5-6 the virus becomes widespread and prevalent among humans. Phases 4-6 clearly signal the need for response, prevention, and control measures. During the post-peak period, pandemic activity drops, but there are still possibilities of recurrent outbreaks, before levels finally return to those seen in seasonal influenza ${ }^{3}$ periods.

These plans from the WHO were made mainly based on the threat levels from the highly pathogenic avian influenza $(\mathrm{H} 5 \mathrm{~N} 1)$, which are much different from the

\footnotetext{
${ }^{2}$ WHO. Pandemic influenza preparedness and response. http://www.who.int/influenza/resources/ documents/pandemic_guidance_04_2009/en/.

${ }^{3}$ WHO. Current WHO phase of pandemic alter for pandemic (H1N1) 2009. http://www.who.int/ $\mathrm{csr} / \mathrm{disease/swineflu/phase/en/index.html.}$
} 
threats posed by Influenza A (H1N1) in 2009, and which are not likely to be the same as future influenza threat levels. These documents have nevertheless played a crucial role in pandemic response efforts and have provided some basic guidance that can be utilized in the outbreak of any infectious disease. The Pandemic influenza preparedness and response also summarized the lessons learned from coping with SARS and the highly pathogenic avian influenza, which will be a great asset in responding to future outbreaks of infectious diseases.

\subsubsection{Assessing Global Laboratory Diagnostic Capabilities for Influenza A (H1N1)}

On May 2nd, 2009, the WHO published its first ever list of countries and laboratories with the capacity to perform PCR (polymerase chain reaction) testing used to diagnose the Influenza A (H1N1) virus in humans, which was updated and re-published on May 4th, 2009. The WHO's criteria for diagnostic capabilities are: "Scoring $100 \%$ in the last two or more WHO external quality assurance programme panels (EQAP) received by the laboratory; or scoring $100 \%$ in the last panel and having a history of consistent results for earlier panels." On the list published were 98 institutions in 73 countries which were able to perform PCR to diagnose the Influenza A (H1N1) virus in humans.

\subsubsection{Pandemic Alert and Risk Assessment}

\subsubsection{Revising Alert Levels}

In response to the outbreak and spread of Influenza A, in the initial stages of the pandemic, the WHO began working on various alert and preparedness plans. On April 25th, 2009, the WHO held an emergency meeting, swiftly determining the severity of the pandemic situation and announced that it constituted a public health emergency of international concern. ${ }^{4}$ On the evening of April 27th, 2009, the WHO raised the influenza pandemic alert level from Phase 3 to Phase $4,{ }^{5}$ and again to Phase 5 on the evening of April 29th. ${ }^{6}$ Then in May during the World Health Assembly, it called on the international community to stay alert. On June 11th, the

\footnotetext{
${ }^{4}$ Statement by WHO Director-General Margaret Chan. Swine influenza. 25 April 2009, http:// www.who.int/mediacentre/news/statements/2009/h1n1_20090427/en/index.html.

${ }^{5}$ Statement by WHO Director-General Margaret Chan. Swine influenza. 27 April 2009, http:// www.who.int/mediacentre/news/statements/2009/h1n1_20090427/en/index.html.

${ }^{6}$ Statement by WHO Director-General Margaret Chan. Swine influenza. 29 April 2009, http:// www.who.int/mediacentre/news/statements/2009/h1n1_20090427/en/index.html.
} 
level was raised to Phase 6, the highest level the WHO has declared in the past 41 years - signalling the onset of a global influenza pandemic. On August 10th, 2010, based on its global assessment, the WHO removed the Phase 6 alert level and announced that the world was moving into the post-pandemic period. ${ }^{7}$

While adjusting pandemic alert levels, the WHO proposed that countries stay flexible in tailoring their specific response measures to their local epidemic situations, and warned that Influenza A (H1N1), as highly infectious as it is, would continue to do harm in the infected countries and could potentially spread to more countries. As the virus continued to spread in the southern hemisphere, which was at that time entering winter, the risk of its combination and mutation with other local epidemic influenza viruses increased, and so the international community was still required to closely monitor the situation.

\subsubsection{Assessing the Risk of the Influenza A (H1N1) Pandemic}

In the early days of the pandemic, the WHO's Influenza Pandemic Assessment Team published its assessment results on May 11th, 2009, in which a comparison was made with the 1957 and 1918 pandemics. ${ }^{8}$ The assessment came to the following conclusions: this was a new subtype of the Influenza A virus; the Influenza A (H1N1) virus was likely to become more contagious than seasonable influenza viruses; differences in clinical symptoms were related to the patient's overall health situation; young people were more susceptible to the virus; the mortality rate was expected to be far lower than the 1918 pandemic; and there were still many uncertainties surrounding the pandemic.

After the pandemic tapered off, on April 12th, 2010 the International Health Regulations Review Committee held its first meeting in Geneva to assess the global response and the functioning of the IHR in relation to the pandemic, as well as to summarize related experiences and lessons learned. The assessment work is still under way and completion is expected in May 2011. ${ }^{9}$

\subsubsection{Response Measures}

In addition to its preparation and alert efforts, the WHO also strengthened pandemic monitoring and introduced a series of strategies and measures relating to pandemic response, treatment, vaccine development, inoculation, and distribution.

\footnotetext{
${ }^{7}$ Director-General's opening statement at virtual press conference. H1N1 in post-pandemic period. 10 August 2010. http://www.who.int/mediacentre/news/statements/2010/h1n1_vpc_20100810/en/ index.html.

${ }^{8}$ WHO (2009b).

${ }^{9}$ WHO. International Health Regulations (IHR) Review Committee. External review of pandemic response. http://www.who.int/ihr/review_committee/en/index.html.
} 


\subsubsection{Integrating Global Pandemic Information}

Beginning on April 24th, 2009, when it first published information on the outbreak of human swine influenza in the U.S. and Mexico, the WHO continually released pandemic and epidemiological information to the globe with the intention of facilitating international communication and sharing.

From April 24th through July 6th, 2009, during the early days of the pandemic, every day or every other day, the WHO published new laboratory-confirmed cases and deaths in affected countries, and at the same time it closely tracked the global transmission of Influenza A (H1N1).

\subsubsection{Adjusting Pandemic Monitoring Methods}

As the pandemic developed, WHO experts considered that as far as pandemic risk monitoring and response strategies were concerned, continued laboratory virus testing to all patients was no longer necessary, as it could overburden laboratories and thus influence their capacity in caring for critically ill patients and other unusual circumstances. ${ }^{10}$ On July 16th, 2009, the WHO announced that countries affected by the epidemic were no longer required to report new confirmed cases, and recommended that attention be placed on monitoring influenza viruses and unusual epidemic events. But countries where Influenza A was not present still needed to report cases as they were discovered.

After April 2010, although the increasing rates of the fatality were on the decline and the pandemic activity remained relatively low, the WHO continued the monitoring of the pandemic and remained in close contact with public health experts in countries across the globe in order to determine whether the virus activity had returned to levels and patterns normally seen for seasonal influenza. Global pandemic activity had remained low over the past few months, and there was little evidence of higher pandemic influenza activity than what was normally caused by the seasonal influenza. The transmission of the Influenza A virus still persisted in the Southern Hemisphere, but it was still impossible to determine if countries there had transitioned to levels and patterns expected for seasonal influenza. Therefore, the WHO continued conducting epidemiological monitoring of the global pandemic situation and reported on relevant information. ${ }^{11}$

\footnotetext{
${ }^{10}$ Zhang (2009).

${ }^{11}$ WHO. Monitoring patterns and levels of worldwide activity. http://www.who.int/csr/disease/ swineflu/notes/briefing_20100721/en/index.html.
} 


\subsubsection{Issuing Technical Guidance on Influenza Prevention and Control}

On April 25th, 2009, the WHO released its first Viral Gene Sequences to Assist Update Diagnostics for Influenza A (H1N1). By August 2010, the WHO had published more than 60 guidance documents on public health topics related to the influenza pandemic, including clinical care, medical treatment and management, laboratory and etiological detection, alert and response, surveillance, epidemiology, vaccines, travel, and personal protection. ${ }^{12}$

In the early days of the Influenza A Pandemic, the WHO's guidance documents were primarily about pandemic preparedness and responses as well as laboratory and hospital management. On May 6th, 2009, the WHO published a guidance document on biorisk management for laboratories handling specimens of Influenza A that were suspected or confirmed to have caused the pandemic, including recommendations of checklists for laboratory managers and staff members as well as minimum operating conditions for special related laboratory operations. ${ }^{13}$ In addition, the WHO also published the Case management of Influenza A (H1N1) in air transport, ${ }^{14}$ the Clean hands protect against infection, ${ }^{15}$ and the Clinical management of human infection with pandemic (H1N1) 2009, ${ }^{16}$ all of which provided valuable technical guidance in the early stages of the pandemic for containing its transmission and treating those infected.

With the spread of the virus along with a growing understanding of it, the WHO revised previously published guidance documents such as the Clinical Management of Human Infection with Pandemic (H1N1), and the Laboratory Diagnosis of Influenza A (H1N1). Published on May 21st, 2009, the Clinical Management of Human Infection with Pandemic (H1N1), was formulated based upon updated virus information as well as data relating to seasonal influenza and the highly pathogenic avian influenza because at that time there was little case data available on H1N1. As experts and researchers began to learn more about the virus and evidence mounted for possible treatments, in October 2009, through a panel of experts in public

\footnotetext{
${ }^{12}$ WHO. Guidance documents on pandemic (H1N1) 2009. http://www.who.int/csr/disease/ swineflu/guidance/en/index.html.

${ }^{13}$ WHO. Laboratory biorisk management for laboratories handling pandemic influenza A (H1N1) 2009 virus http://www.who.int/entity/csr/resources/publications/swineflu/Laboratorybio riskmanagement.pdf.

${ }^{14}$ WHO. Case management of Influenza A (H1N1) in air transport. http://www.who.int/csr/ resources/publications/swineflu/air_transport/en/index.html.

${ }^{15}$ WHO. Clean hands protect against infection. http://www.who.int/csr/resources/publications/ swineflu/AH1N1_clean_hands/en/index.html.

${ }^{16}$ WHO. Clinical management of human infection with pandemic (H1N1) 2009. http://www.who. int/csr/resources/publications/swineflu/clinical_management/zh/index.html.
} 
health, laboratory, pathological and clinical fields, the WHO published their revised guidance in November 2009, ${ }^{17}$ providing updated technical support for the diagnosis and treatment of patients with Influenza A (H1N1).

\subsubsection{Coordinating Vaccine Research and Development}

After the outbreak of Influenza A (H1N1), the WHO consulted related pharmaceutical manufacturers about developing vaccines, encouraging worldwide support of Influenza A (H1N1) vaccination production. The organization also collaborated with drug authorities in related countries ensuring that newly developed Influenza A vaccines met as many safety standards as possible. Meanwhile, the organization helped China in efficiently obtaining live strains of the Influenza A (H1N1) virus, which accelerated the country's research and development of relevant vaccines and drugs.

While ensuring an adequate amount of seasonal influenza vaccines were available, the WHO also initiated research and development for Influenza A (H1N1) vaccinations in the early stages of the pandemic. ${ }^{18}$ Given that global limited production capacity for antiviral drugs and influenza vaccines could never meet the healthcare needs of 6.8 billion people, the WHO recommended governments to have clear and targeted prevention and control measures to avoid waste of resources. $^{19}$

\subsubsection{Planning for Vaccination Distribution}

On July 2nd, 2009, a meeting of the world's health ministers was held in Mexico to assess the influenza pandemic and discuss countermeasures and inoculation distribution. At the meeting, WHO Director-General Margaret Chan called for international collaboration and solidarity, while stressing that special attention must be paid to high-risk groups like pregnant women and patients with chronic diseases. The WHO also called on vaccine manufacturers to provide them a certain amount of free vaccines so as to help developing countries better cope with their epidemics. $^{20}$

In response to the ongoing global pandemic, the WHO stressed the importance for countries to carry out inoculations and to set forth three goals for their vaccination strategies, i.e. ensuring the normal operation of national healthcare systems,

\footnotetext{
${ }^{17}$ WHO. Clinical management of human infection with pandemic (H1N1) 2009: revised guidance. http://www.who.int/csr/resources/publications/swineflu/clinical_management/en/index.html.

${ }^{18}$ WHO Says Old Vaccines Production and New Vaccines Development Equally Important. Ecns. cn, May 16, 2009. http://world.people.com.cn/GB/9311296.html.

${ }^{19}$ Liu and Yang (2009).

${ }^{20}$ World Health Ministers' Meeting Discusses Measures against Influenza A (H1N1). Xinhuanet.com, July 4, 2009. http://medicine.people.com.cn/GB/9593164.html.
} 
lowering morbidity and mortality, and minimizing possibilities of community-level outbreaks. To ensure continued normal operations of healthcare systems, the WHO recommended medical workers first be vaccinated, then pregnant women, patients aged six months and older with such chronic illnesses like asthma and obesity, healthy people aged 15-49, healthy children, healthy people aged 50-64, and people aged 65 and older - in that exact order. ${ }^{21}$ The WHO also urged pharmaceutical manufacturers to produce vaccines at full capacity, to ensure fair distribution among developed and developing countries. Countries such as China, Italy, France, the United States, Germany, the United Kingdom, Norway, Sweden, Finland, Australia, and Japan took steps to vaccinate domestic residents, based on their own epidemic situations, healthcare resources, and ability to acquire vaccines. Some of the countries placed orders for more vaccines in order to cope with potential outbreaks. $^{22,23}$

Response strategies varied widely across countries (see a detailed description in the next section) because each was faced with outbreaks and developments with different characteristics, in addition to political, economic, and cultural dissimilarities, especially in their public health systems which varied in both management and operation. While developed countries already had fairly effective response measures in place thanks to their advanced economic and social development as well as robust healthcare systems, some developing countries with poor economic foundations and weak public healthcare had a much harder time dealing with public emergencies. Therefore, they had an even harder time in dealing with Influenza A (H1N1). After the pandemic broke out, countries showed varied responses to the WHO's recommended response strategies and measures; in particular developing countries that had greater reliance on these strategies and measures as well as technical assistance from the WHO, were much more proactive. There is no doubt that the WHO played a crucial role in helping countries worldwide-especially developing ones - in coping with the pandemic, whether it is pandemic monitoring, clinical diagnosis and treatment of the virus, or vaccine development and distribution. However, because this pandemic originated in North American countries, taking into account the political, economic and cultural differences between countries as well as their different response capabilities, the WHO was also faced with new challenges like how to provide tailored guidance to developed and developing countries. The purpose of this guidance was to increase the effectiveness of related strategies and measures, mitigate and contain the spread of the pandemic, and minimize the negative effects of the virus on society and populations. Such targeted guidance was not particularly prevalent in their guidance regarding

\footnotetext{
${ }^{21}$ Influenza A (H1N1) Is More Pathogenic Than Comman Flu, WHO Deems Vaccination Necessary. People.cn, July 15, 2009. http://medicine.people.com.cn/GB/9654341.html.

${ }^{22}$ Sweden Begins Vaccinating Its People against Influenza A (H1N1) in Autumn. Xinhuanet.com, July 15, 2009. http://medicine.people.com.cn/GB/9658730.html.

${ }^{23}$ Developed Countries Rush to Buy Influenza A (H1N1) Vaccines, Leaving Poor Countries Worried about Epidemic Control. People.cn, July 28, 2009. http://medicine.people.com.cn/GB/ 9732375.html.
} 
response strategies and measures as the requirements placed on developed countries were quite low, resulting in an overall devaluation of said proposed strategies and measures. Therefore, when confronting similar public health emergencies in the future, the WHO should present more pertinent strategies and tailored measures which could play greater roles in pandemic preparation and response.

\subsection{National and Regional Response Strategies and Measures}

The outbreak in late April 2009 of Influenza A (H1N1) in several North American countries quickly attracted attention in related countries. Responding promptly to the crisis, government agencies and related departments in multiple countries immediately initiated public health emergency mechanisms and put into action a wide range of prevention and control strategies and measures.

\subsubsection{National and Regional Influenza Response Systems and Mechanisms}

\subsubsection{National Response Mechanisms for Influenza A (H1N1)}

Considering the serious economic, social, and public health consequences that could happen due to the outbreak, coping with the pandemic would demand participation, coordinated preparation, and enhanced collaboration from governments and different departments. Some countries specifically established unified leadership bodies and related mechanisms to deal with the pandemic, while others did so through existing government bodies or departments.

For example, countries like the United Kingdom, India, Japan, and Mexico set up a special coordination and management mechanism, and established an emergency decision-making, command and coordination body which was directed by the heads of government with the guidance and participation of relevant agencies. The British government specifically established a ministerial committee consisting of related government departments to strengthen inter-departmental communication and coordination and ensure the formulation and execution of preparation and response policies. The Indian Ministry of Health and Family Welfare established the Inter-Ministerial Task Force and Joint Monitoring Group for AI/pandemic to direct and coordinate the national response to the pandemic. ${ }^{24}$ France's public health emergency mechanism was run by the "Inter-ministerial Risk Group" with

\footnotetext{
${ }^{24}$ Dr. Shashi Khare. Pandemic influenza A H1N1: Preparedness \& response in India. CDC New Delhi. http://209.61.208.233/LinkFiles/RCE_DAY02_H1N1_INDIA-Dr_Shashi_Khare.pps.
} 
the responsibility of decision making, situational tracking, and publicity, and the Minister of the Interior acted as the lead and was responsible for approving and initiating such decisions. Japan established the New Influenza Response Headquarters directed by the Prime Minister, and transformed the Risk Management Center's Information Liaison Office under the Prime Minister's Official Residence into the Official Residence's Liaison Office for directing and coordinating national pandemic response efforts. Mexico, whom in the past responded to public health emergencies mainly through direct government interventions and temporary emergency groups, established the National Committee for Health Security (CNHS) for analyzing, monitoring, and assessing the security issues of national health policies and for proposing relevant policies.

The United States, Australia and some other countries didn't specifically establish a governing body in response to the pandemic. After its incorporation in 2003 into the United States Department of Homeland Security (DHS), the Federal Emergency Management Agency's (FEMA's) responsibilities were expanded from natural disaster response to counter terrorism and pandemic diseases. The FEMA Director, appointed by the President, reports directly to the Secretary of Homeland Security and may, in response to a crisis, be summoned by the President to attend ministerial-level meetings and take part in the decision-making process. After the influenza pandemic outbreak in 2009, the United States launched its standard emergency response procedures, which included close collaboration and coordination among the federal, state and local governments along with the private sector. The U.S. Congress was charged mainly with funding public health efforts at the federal, state, and local levels, ${ }^{25}$ while it was the responsibility of the federal government to update response plans, strengthen the development and revision of community-based plans, and enhance response capabilities. The DHS oversaw the distribution of antiviral medications and the dissemination of pandemic information to the public. ${ }^{26}$ The U.S. Department of Health and Human Services (HHS), the executive body of pandemic preparation and response, was in charge of deploying, directing, and overseeing various response efforts, and they also completed the following: issued guidance on the influenza pandemic, ${ }^{27}$ provided technical, financial, and medical support to states, and based on pandemic analysis announced a national state of emergency. As the national public health institute under the HHS, the Center for Disease Control and Prevention (CDC) played a crucial role in virus monitoring, prevention, and control. Similarly, Australia established a mechanism in which an inter-agency committee under the leadership of the prime minister and the cabinet was in charge of determining the federal government's preparation and

\footnotetext{
${ }^{25}$ Weissman (2009).

${ }^{26}$ Department of Homeland Security. Testimony of Alex Garza, MD, MPH, Office of Health Affairs, before the Senate Homeland Security and Governmental Affairs Committee on "H1N1 Flu: Getting the Vaccine to Where it is Most Needed". November 17, 2009. http://www.dhs.gov/ ynews/testimony/testimony_1258473176155.shtm.

${ }^{27}$ Craig Vanderwagen (2009).
} 
response strategies as well as pandemic countermeasures, ${ }^{28}$ with state governments making and implementing relevant policies under the guidance of the federal government.

Whether or not a governing body was established for management of the pandemic, countries worldwide attached great importance to collaboration among government institutes and departments. For example, interim pandemic assessment reports by U.S. departments all mentioned that the timely response to, and rapid progress made in coping with the influenza pandemic, were due in large part to the clear divisions of labor and close collaboration among federal government institutes, departments, and state, and local governments. ${ }^{29,30}$ The Indian government also stressed that pandemic responsibilities did not fall solely on the health department, and that it was necessary for multiple departments to collaborate with one another; the following departments of India were involved in pandemic preparation and response: the Ministry of Finance which provided cash, budgets, risk management, and insurance; the Ministry of Commerce and Industry which provided medical equipment; the Ministry of Road Transport and Highways which was charged with handling relevant transportation and communication issues; the Ministry of Defense and related military departments which was charged with public services, laws and regulations, security, and human rights; the Ministry of Information and Broadcasting which guaranteed the transparency of strategic communication, the dissemination of information, etc.; the Ministry of Environment and Forests and the Ministry of Health and Family Welfare which ensured biosafety, sanitation, wildlife conservation, etc.

\subsubsection{Examples of National Pandemic Response Strategies (Plans)}

To effectively curb the transmission of the pandemic and its negative effects on society, many countries formulated a national strategy or plan against possible influenza outbreaks from 2003-2005, outlining the duties and division of labor among government departments as well as their preparation and response strategies. Their policies on Influenza A (H1N1) were generally built on these strategies.

In 2005, pursuant to the Pandemic Preparedness Guidance published by the WHO, the United States developed the HHS Pandemic Influenza Plan and the National Strategy for Pandemic Influenza, according to which preparation and response strategies and measures would be chosen based upon phases that measured the pandemic's development. Included in the documents are detailed provisions about the duties along with preparation and response strategies of related government departments and mechanisms, i.e.: inter-departmental collaboration,

\footnotetext{
${ }^{28}$ Council of Australian Governments/Working Group on Australian Influenza Pandemic Prevention and Preparedness. National Action Plan for Human Influenza Pandemic. 2010.

${ }^{29}$ Sebellus (2009a).

${ }^{30}$ Sebellus (2009b).
} 
public risk communication, vaccine production and distribution, and the stockpiling of antiviral medications.

In accordance with the WHO Pandemic Preparedness Guidance, the United Kingdom published their Influenza Pandemic Contingency Plan in 2005, and their National Framework for Responding to an Influenza Pandemic in 2007, which stipulated that strategies and measures for both preparation and response would be selected based upon pandemic phases.

In 2005, Australia formulated the Australian Heath Management plan for Pandemic Influenza and later revised it in 2008, and it remains as the country's national-level health plan for an influenza pandemic. ${ }^{31}$

India formulated the Influenza Pandemic Preparedness and Response Plan in 2005, which was used as a foundation for prevention and control policies against Influenza A (H1N1).

In 2006, the Mexican government issued the National Preparedness and Response Plan for Pandemic Influenza, ${ }^{32}$ on which the country's prevention and control policies against influenza A were built.

On May 11th, 2009, the Japanese government swiftly issued the Action Plan for Measures against Influenza A (HINI) to curb its domestic transmission. This plan contained response measures formulated according to four phases of distinct pandemic phases, i.e. occurrence overseas, early occurrence at home, infection expansion-spread-recovery, and stabilization.

\subsubsection{National Funding for Influenza Pandemic Prevention and Control}

For countries across the globe, central governments primarily provided the funds for prevention and control efforts against Influenza A (H1N1), and these funds were made available to related departments in the different pandemic phases. During the initial period and at the peak of the pandemic, these funds were mainly used for stockpiling antiviral drugs; purchasing relevant equipment, facilities, protective supplies and other materials; establishing points of distribution for antiviral drugs; providing patients with free antiviral drugs; and carrying out pandemic monitoring. During post-peak periods, funds were mainly utilized to purchase unified Influenza A vaccines from manufacturers, which were then distributed to the public with no charge. ${ }^{33}$ Some developed countries also specifically established foreign assistance funds that provided developing countries both monetary and material assistance in combatting the pandemic.

\footnotetext{
${ }^{31}$ Australian Health Management Plan for Pandemic Influenza. http://www.health.gov.au/internet/ panflu/publishing.nsf/Content/ahmppi-2009.

${ }^{32}$ Plan Nacional de Preparación y Respuesta ante una Pandemia de Influenza. http://www.dgepi. salud.gob.mx/pandemia/FLU-aviar-PNPRAPI.htm.

${ }^{33}$ DH. Pandemic H1N1 (2009) Influenza: Chief Medical Officer's Fortnightly Bulletin for Journalists. 21 January 2010.
} 
The United States Congress invested heavily in pandemic prevention and control. In 2006, the Congress provided an appropriation of more than seven billion U.S. dollars (USD) for implementing the pandemic preparedness strategy. On April 28th, 2009, the U.S. President received another appropriation of 1.5 billion USD from Congress which was specifically designated for combatting the swine flu. In July of that year, Congress provided 1.85 billion USD to be used as funds for emergency resource deployment and an additional 5.8 billion USD for emergency preparation and response against the influenza pandemic. ${ }^{34}$ In September, the Congress went on to make 1444 million USD available to states and hospitals for carrying out vaccination programs. ${ }^{35}$ Meanwhile, the United States Agency for International Development (USAID) provided Mexico with five million USD in emergency aid funds, 900,000 sets of personal protective equipment for virus monitoring personnel, and Tamiflu for 400,000 courses of treatment. Additionally, the HHS provided 147 countries with 769 laboratory diagnostic kits, and donated to the Pan American Health Organization (PAHO) medications for 420,000 courses of treatment in aid of Latin American and Caribbean countries.

In Australia, funds for prevention and control against Influenza A (H1N1) originated mainly from the federal government, which was used specifically for monitoring pandemic development, stockpiling and distributing antiviral drugs, training medical personnel, providing free vaccinations for citizens, and assisting developing countries with prevention and control efforts. The federal government spent 43 million USD on antiviral drugs, 1.4 million USD on the purchasing of automatic detection equipment for the National Influenza Center and other public health laboratories, 4 million USD on training general practitioners across the country, and 3 million USD on a donation to the WHO which was used in aiding developing countries, especially those neighboring Australia, with pandemic monitoring, detection, preparation and response.

In the United Kingdom, funds for responding to Influenza A (H1N1) came mainly from the British government; by January 20th, 2010, the Department of Health had dispensed to the nation 1.26 million doses of Pandemrix, an influenza vaccine developed by GlaxoSmithKline, and 370,000 doses of a Baxter-developed vaccines. ${ }^{36}$

The Indian government established a one billion rupee disaster response fund in accordance with the Disaster Management Act, which was administered by the Ministry of Home Affairs, and this disaster fund accepted donations from individuals and organizations. In addition, a national disaster fund was specifically established to finance disaster relief and recovery efforts. State governments also

\footnotetext{
${ }^{34}$ William Corr (2009).

${ }^{35}$ See Footnote 29.

${ }^{36}$ DH. Pandemic H1N1 (2009) Influenza: Chief Medical Officer's Fortnightly Bulletin for Journalists. 21 January 2010.
} 
established disaster response funds and relief funds in accordance with the law at the state and regional levels. ${ }^{37}$

Mexico invested a total of 350 million USD in Influenza A (H1N1) preparation and response, including the purchasing of drugs and vaccines, and the adoption of other prevention and control efforts. ${ }^{38}$

\subsubsection{National Prevention and Control Policies and Measures}

\subsubsection{Outbreak of the Influenza Pandemic}

\section{Pandemic Outbreak Alert}

After the outbreak of the Influenza A (H1N1) Pandemic, some countries, in accordance with WHO pandemic alert phases as well as their domestic situations, initiated and adjusted their pandemic alert levels. The United States, for example, declared a public health emergency on April 26th, 2009, and made several updates afterwards $^{39}$ until the stage of emergency was lifted on June 23rd, 2010. ${ }^{40}$ Declaring a state of emergency helped the HHS prepare for and respond to the influenza pandemic, and prompted the Food and Drug Administration (FDA) to issue Emergency Use Authorizations (EUAs) for the use of antiviral drugs and therapeutic tools - i.e. they approved the use of Relenza and Tamiflu as stockpiled antiviral drugs for prevention and control of the virus, RT-PCR for virus detection, and N95 masks, which protected pandemic-affected communities. On April 29th, 2009 , in light of the WHO's pandemic alert phases and its national pandemic situation, Singapore raised their alert level in its five-level disease warning system from Green to Yellow, and again to Orange the next day.

\section{Inspection and Quarantine}

To prevent the influenza virus from spreading into and circulating within their territories, many countries adopted strict inspection and quarantine measures in the early days of the pandemic. The United States screened travelers from Mexico and other countries, conducting both temperature and medical inspections. Cargo-especially

\footnotetext{
${ }^{37}$ Xiaoming (2009).

${ }^{38}$ Mexico Announces Pandemic Influenza Alert Removed. http://www.chinadaily.com.cn/microreading/mfeed/hotwords/20100811734.html.

${ }^{39}$ See Footnote 34.

${ }^{40}$ HHS declares public health emergency for swine flu. April 26, 2009. http://www.hhs.gov/news/ press/2009pres/04/20090426a.html.
} 
baggage and raw meat products from epidemic affected areas - were strictly quarantined; many airlines required their service staff to observe and question passengers suspected of illness, and when necessary, have them examined. American border officials between the United States and Mexico also were required to examine the physical condition of travelers crossing the border and be prepared to take necessary measures. Additionally, citizens were asked to stop all unnecessary travel into epidemic areas.

Australia also implemented strict border control, requiring all flights from the Americas to report the health status of passengers on board before landing; any individual with influenza-like symptoms had to be assessed by Australian quarantine authorities in order to determine if further treatment was required; eight major airports across the country were equipped with body temperature measuring instruments, and every incoming passenger was required to complete a health declaration card.

India adopted pandemic monitoring measures at airports, sea ports, and inland ports across the country; all incoming passengers to the twenty two international airports were screened, especially those from epidemic areas or with influenza symptoms, who were then quarantined and treated for at least three days. Medical personnel were trained in advance, and were required to wear masks, gloves, and protective clothing at work. Influenza A (H1N1) inspection standards and operational rules were formulated and implemented national widely at that time.

Japan's Ministry of Health, Labor and Welfare required all flights from Mexico, United States, and Canada arriving at the Narita, Kansai and Chūbu Centrair International Airports be inspected while aboard the plane. Local airports not included on the list of airports for quarantine measures, for example in Niigata, Akita and Hiroshima, also decided to follow suit and expanded the scope of quarantine to include flights from South Korea, Hong Kong, and some other countries and regions. Japanese border inspection and quarantine authorities screened people from Mexico, the United States and had cargo strictly quarantined, especially baggage and raw meat products from epidemic areas.

\section{Preparedness}

While applying strict control measures against the importation of the virus, in the early days of the pandemic countries also began strengthening preparation capacity building. For example, in the United States, during the initial stage of the outbreak, the HHS dispensed medication from the Strategic National Stockpile enough to treat three million people, the Department of Defense (DOD) separately readied enough medication for seven million soldiers, and the CDC allocated antiviral drugs, protective equipment, and testing kits. At the same time, the HHS provided training for medical personnel with the goal of enhancing their abilities in treating and handling the pandemic. The German government required each state to stockpile enough antiviral drugs to use for $20 \%$ of their populations. South Korea increased budget spending so that by the end of October the country's had enough 
drugs stored for $10 \%$ of its population. In an effort to mitigate the spread of the virus, the Indian government designated specific hospitals to treat Influenza A (H1N1) cases.

\section{Health Education}

To increase public awareness of the pandemic, countries developed large scale health education and communication projects. The U.S. CDC provided health recommendations to society, communities, clinical workers and other professionals, and launched an online live-broadcast health education program, "Know What to Do about the Flu," to help strengthen the public's abilities in protecting themselves against the virus. The United Kingdom updated pandemic situations and work priorities on a regular basis via an official government website, and provided technical support relating to virus prevention and treatment. India published a "public notice' through national media channels with the aim of disseminating knowledge and increasing public awareness of Influenza A (H1N1) prevention and control. The government also set up a toll-free service hotline to answer questions about the influenza pandemic. In Japan, an information, education and communication campaign was launched targeting high-risk groups of people arriving at and departing from the country's international airports, and the Ministry of Health, Labour and Welfare opened an information window to answer questions from the public.

\subsubsection{The Spread of the Influenza Pandemic}

\section{Focusing on Clinical Treatment}

As the pandemic developed and more cases emerged, it was found that the majority of cases were coming from local communities instead of from abroad. At this point in time, the continued use of containment strategies had been ineffective, and medical personnel were having to dedicate more time and energy to the increasing number of patients.

According to the National Response Framework, the HHS in the United States needed to stockpile enough antiviral drugs for one-fourth of the country's population during the pandemic, and to prepare at least six million treatment courses during the pandemic's initial phase. In the spring of 2009, the HHS allocated eleven million treatment courses that could be used for rapid response against the pandemic. The CDC and the FDA also worked together to address potential options for treatment of severely hospitalized patients. ${ }^{41}$ In October, the HHS shipped an additional 300,000 bottles of the antiviral oseltamivir in oral suspension formula to

\footnotetext{
${ }^{41}$ Anna Schuchat (2009a).
} 
states in order to mitigate a predicted national shortage. The FDA worked closely with the CDC, the Office of the Assistant Secretary for Preparedness and Response (ASPR), manufacturers, and others to increase production and availability of personal protective equipment such as gloves, masks, and respirators. At the same time, the 2009 Influenza (H1N1) Consumer Protection Team, established by the FDA, put in place an aggressive strategy to combat fraudulent influenza products. ${ }^{42}$

The British Secretary of State for Health declared on July 2nd, 2009, that the United Kingdom's response efforts were transitioning from a "containment phase" to a "treatment phase." In order to cure patients more efficiently, the British government created a national stockpile by the purchasing of more antiviral drugs, and drug distribution centers were also established across the country, with the National Health Service (NHS) playing a leading role in treatment provisions. To relieve pressure on medical institutions, on July 23rd, 2009, the British government launched the National Pandemic Flu Service (NPFS). ${ }^{43}$ The NPFS was a self-help healthcare system which, through a dedicated website and call centers, provided people worried about flu-like symptoms with professional assessment services, including the suggestions on whether they should receive treatment or contact a general practitioner, etc. A person, if assessed as indeed having Influenza A (H1N1) symptoms, would be given an authorization number by the system, which he or she could use to pick up antiviral drugs from one of local distributions centers. The launch of this system effectively mitigated the pressure on primary healthcare institutions and allowed general practitioners to dedicate their attention to critically ill patients.

In order to quickly detect and treat critically ill patients, and also to ensure an adequate number of hospital beds as the number of cases increased, the Japanese government readjusted its guidelines on pandemic response efforts, and discarded the practice of classifying regions according to rate of transmission in that area. According to the revised guidelines, regular hospitals received patients infected with Influenza A (H1N1); all mildly ill patients were instructed to medicate and rest at home, rather than being hospitalized. For patients with asthma or other illnesses whom had contracted Influenza A (H1N1) and whose conditions were likely to worsen, a PCR (polymerase chain reaction) test or other Influenza A virus test was performed, and effective antiviral drugs were administered as early as possible. When necessary, decisions would be made to get them hospitalized. Japan gradually used the confirmed cases reported from a certain number of hospitals as estimations and predictions for that area's infected population.

Australia used antiviral drugs from their national medical stockpile to treat moderately and critically ill patients, especially those with severe breathing difficulties or those whose conditions were rapidly worsening. All medical personnel, who contracted Influenza A (H1N1) and developed moderate symptoms of

\footnotetext{
${ }^{42}$ Jesse Goodman (2009).

${ }^{43} \mathrm{DH}$. Launch of the National Pandemic Flu Service. http://webarchive.nationalarchives.gov.uk/+/ www.dh.gov.uk/en/Publichealth/Flu/Swineflu/DH_102909.
} 
infection, or were more prone to develop serious symptoms, were eligible for antiviral treatment. Patients with fairly mild symptoms were encouraged to self-medicate.

India issued clinical management guidelines, where the Indian Committee on Infectious Diseases published guidance on the screening and clinical treatment of laboratory-diagnosed cases of Influenza A (H1N1); the Ministry of Health and Family Welfare issued guidelines on family isolation, clinical examinations, and hospitalization by categories of Influenza A (H1N1) cases-where Categories A/B patients were asked to be isolated and reduce contact with their family and others and Category $\mathrm{C}$ patients required immediate hospitalization. All suspected cases were tested at the National Institute of Communicable Diseases (NICD) in New Delhi, or at the National Institute of Virology in Pune, and then examined further at relevant laboratories. India currently has forty four laboratories dedicated to the early management of controlling confirmed cases. ${ }^{44}$

\section{Policies for Community-based Non-drug Pandemic Mitigation}

Given the dynamic nature of the pandemic, involving each and every citizen in its mitigation became a very important part of global response efforts.

To contain the pandemic, Mexico mobilized a large force of police officers and soldiers to execute the following: distribute masks among citizens for free, shut down public places, cancel or delay large-scale events, halt teaching activities in all schools - including universities, primary and secondary schools, and kindergartens -in Mexico City and in the State of Mexico. On April 29th, 2009, the Mexican government declared a suspension of all nonessential public affairs and economic activities from May 1st through May 5th. Moreover, the Mexican government also adopted a wide range of measures to strengthen pandemic information communication and sharing, i.e.: reporting pandemic developments via media channels, setting up 800 hotlines, launching influenza prevention websites, giving out leaflets on pandemic information that called for personal hygiene and increased public awareness of the virus. ${ }^{45}$

In the United States, the HSS launched a one-stop influenza information website (www.flu.gov), which gathered information from regular media briefings conducted by the HHS and other federal agencies, ${ }^{46}$ and provided the public with scientific and effective information services. In collaboration with federal, state, and local partners, the HHS also developed a wide range of community-based intervention guidelines which were being evaluated simultaneously. The CDC and the DHS provided specific recommendations targeted to a wide variety of groups, including the general public, people with certain underlying health conditions, infants, children, parents,

\footnotetext{
${ }^{44}$ John and Moorthy (2010).

${ }^{45}$ Del Rio and Hernandez-Avila (2009).

${ }^{46}$ See Footnote 41.
} 
pregnant women, seniors, health care workers, workers in relevant industries, laboratory workers, and homeless people. With these recommendations, people were equipped to take appropriate action in reducing the transmission of the virus, especially in early autumn before vaccines were widely disseminated. The CDC also provided, and updated on a regular basis, scientific guidance on influenza prevention and control to schools, daycares, universities, large and small businesses, and federal agencies. These comprehensive guidelines provided not only advice on how individuals and institutions could protect themselves against the virus and mitigate its spread, but also recommendations for healthcare providers about the appropriate use of anti-viral drugs, especially in treating patients who were at the highest risk of suffering complications from the influenza. ${ }^{47,48}$

In Japan, after the alert level transitioned from an "overseas pandemic" phase to the heightened "early onset of a domestic pandemic" phase, the local governments of Osaka and Hyōgo Prefectures required the following for areas where infections had occurred: gatherings and collective recreational activities be suspended, entertainment venues be temporarily closed, social service workers be required to wear masks, teaching activities of varying levels at more than one thousand educational institutions be suspended for one week, citizens avoid trips and gatherings, and business activity be reduced for the time being.

In Australia, patients with mild symptoms were allowed to stay at home as a means of isolation.

\section{Revising Pandemic Monitoring}

With the rapid spread of the pandemic, the United States didn't take stock in counting cases, but instead focused on the evolution process of the virus. ${ }^{49}$ The United States' advanced and unique monitoring system for bacteria and viruses uses dynamic and standardized methods to collect data related to virus occurrence, virus developments, and basic medical trends, and employs national demographic data to compute virus incidence and describe its epidemiological characteristics. This system brings together and facilitates cooperation within the $\mathrm{CDC}$, state health authorities, academic partners, hospitals and infection control centers. Moreover, it contains special research platforms, i.e., socio-economic evaluations of disease risk factors, effects of the disease and vaccinations, data on resources for vaccine research and development, and data on approved vaccines.

In Australia, laboratory testing focused on critically ill patients, high-risk groups with severe diseases, and personnel in relevant institutions. Monitoring was also conducted to see if any resistance or mutations of the virus had occurred.

\footnotetext{
${ }^{47}$ See Footnote 29.

${ }^{48}$ See Footnote 41.

${ }^{49}$ United States Becomes Eye of Pandemic Influenza Storm, Puts out But Not Protect against Fire. Ecns.cn, May 19, 2009. http://news.xinhuanet.com/world/2009-05/19/content_11398140.htm.
} 


\section{Vaccine Development and Inoculation}

Understanding that vaccinations were the best means for combatting the virus, countries focused a large amount of resources on vaccination development and inoculation methods.

In its influenza pandemic preparedness and response plan, the HHS in the United States set two objectives for vaccine preparation ${ }^{50}$ : to stockpile twenty million vaccinations for key personnel, and to increase manufacturing capacity to cover the population in the United States, in other words, produce 300 million doses within six months of the pandemic outbreak. Immediately following the outbreak, the National Institute of Allergy and Infectious Diseases (NIAID) subordinate to the U.S. National Institutes of Health (NIH) began its research on the virus and vaccination development. In July 2009, the NIAID initiated a series of clinical trials on the effectiveness of newly developed vaccines. In September, the FDA approved manufacturing for four vaccination types, which were then made available for distribution among the states. The federal government then identified priority groups for vaccination and formulated an inoculation policy. ${ }^{51}$ Starting on October 5th, a national Influenza A (H1N1) voluntary inoculation program begun targeting high-priority groups including pregnant women; people between the ages of 6 months through 24 years of age; people aged 65 years or older with chronic health disorders like asthma, diabetes and heart disease; and healthcare and emergency services personnel. ${ }^{52}$ During the two months that followed, vaccine manufacturers provided 10-20 million vaccination doses each week, an amount which reached roughly 250 million by the end of $2009 .^{53,54}$ According to statistics, the federal government ordered a total of 229 million doses of the vaccine with the plans of vaccinating 158 million people, and in the end 90 million people were actually inoculated.

On October 21st, 2009, the United Kingdom launched its national Influenza A (H1N1) inoculation program. The first phase of the plan provided the vaccine to the high risk population of fourteen million people, including critically ill patients, pregnant women, and healthcare personnel working in hospitals. Soon afterwards, general practitioners across the country began encouraging people with health disorders or immunity problems, and pregnant women to get vaccinated. On December 8th, 2009, the British government went on to include children ages six months to five years old in the vaccination program.

\footnotetext{
${ }^{50}$ See Footnote 30.

${ }^{51}$ See Footnote 41.

${ }^{52}$ See Footnote 30.

${ }^{53}$ President Obama Declares a National Influenza A (H1N1) Emergency. Ecns.cn, October 25, 2009. http://medicine.people.com.cn/GB/10252536.html.

${ }^{54}$ EU Approves First Influenza A (H1N1) Vaccines. CRI Online, September 30, 2009. http://news. sina.com.cn/w/2009-09-30/024718754642.shtml.
} 
In August 2009, Australia approved a national vaccination program and began providing free vaccinations to healthcare workers, pregnant women, and individuals with chronic health disorders who were susceptible to the virus. On September 30th, the Australian government announced that all adults and children aged ten years and older could also receive free vaccinations.

In May 2009, the Mexican government announced an appropriation of 6.2 million USD for the establishment of a dedicated committee composed of 12 authoritative medical experts, and this committee's mission was to mobilize and coordinate research efforts for carrying out etiological, epidemiological, diagnostic reagent and vaccine research relating to Influenza A (H1N1). It was also responsible for providing policy recommendations on pandemic prevention and control and medical treatment options to the government. ${ }^{55}$

In July 2009 Japan began distributing permits authorizing the utilization of Influenza A (H1N1) vaccines, and they also launched a national vaccination program. The first groups to receive it included healthcare personnel, police officers, as well as high-risk groups like pregnant women, patients with chronic diseases, and seniors.

\subsubsection{Post-peak Period}

As Influenza A (H1N1) cases gradually declined, some countries readjusted their pandemic response levels as well as their measures for virus prevention, control, and treatment. Countries set about making summaries and conducting evaluations while continuing their pandemic monitoring and information sharing.

\section{Readjusting Alert Levels and Measures for Virus Prevention and Control}

In 2010, most regions across the globe saw a decline in Influenza A (H1N1) activity, and though in some regions the virus still sustained its intensity (level), the overall virus transmission dropped. Additionally, it was discovered in most cases that the Influenza A virus only caused mild infections, and that its virulence had not increased since it was first reported in April 2009. Effective vaccinations had been in circulation since November 2009. It was for these reasons that the Singaporean Ministry of Health decided on February 12th, 2010, to downgrade its alert level from Yellow to Green.

Beginning in February 2010, the United Kingdom deactivated the National Pandemic Flu Service (NPFS), an act done in line with ensuring the operational response was appropriate to the threat level posed by the virus and also because general practitioners and primary care trusts could now manage the clinical

\footnotetext{
${ }^{55}$ Mexico Sets up Special Committee for Influenza A (H1N1) Research. Xinhuanet.com, May 12, 2009. http://news.xinhuanet.com/world/2009-05/12/content_11357428.htm.
} 
caseload by themselves. ${ }^{56}$ Anyone concerned about flu-like symptoms were advised to contact their doctor for assessment, who could then issue an antiviral authorization voucher if needed. The NPFS would be reactivated should the pandemic virus regained its virulence. Starting on April 1st, 2010, free antiviral medication from the national stockpile was no longer available to patients with Influenza A (H1N1). Normal treatments and prescription charges were reinstated for those suffering from influenza. ${ }^{57}$

In June 2010, the United States declared the end of the public health emergency.

\section{Expanding Vaccination Coverage}

As confirmed cases declined and the spread of the virus continued to slow, the U.S. federal, state, and local health authorities began to readjust their response strategies. In addition to continued efforts in strengthening public health education and inter-agency collaboration, other measures included bolstering the vaccination campaign, ${ }^{58}$ strengthening virus monitoring, and continuing focus on virus mutations.

\section{Commencing Summarized Pandemic Evaluations}

As the pandemic developed in the United States, especially after the wide distribution of vaccinations to the public, some U.S. agencies and institutions evaluated the results of a range of their prevention and control measures. The purpose of these evaluations were to identify problems that existed in the national pandemic response measures, and correct them to better the response in the future. For example, the Institute of Medicine (IOM) Forum on Microbial Threats analyzed the domestic and global impact of the Influenza A (H1N1) pandemic, while the Department of Defense (DOD) and the Global Emerging Infections Surveillance (GEIS) reviewed influenza response programs, including management and planning, monitoring, laboratory research, response capacity, capacity building, collaboration and coordination, guidance, contact lists, contingency plans, and so on. The evaluation report, Sustaining Global Surveillance and Response to Emerging Zoonotic Diseases, analyzed global influenza A (H1N1) detection, reporting, and response systems, and expounded upon shortcomings and challenges surrounding the pandemic. On March 5th, 2010, the University of Pittsburgh Medical Center's

\footnotetext{
${ }^{56}$ Written Ministerial Statement announcing National Pandemic Flu Service to stand down. http:// collections.europarchive.org/tna/20100509080731/, http://dh.gov.uk/en/Publichealth/Flu/Swineflu/ DH_111890.

${ }^{57}$ Pandemic H1N1: stand down of the antiviral distribution arrangements. http://www.dh.gov.uk/ en/Publicationsandstatistics/Lettersandcirculars/Dearcolleagueletters/DH_114769.

${ }^{58}$ The United States Advertises Public Vaccination against Influenza A Virus. Evening News, December 8, 2009. http://news.sina.com.cn/h/2009-12-08/124319215143.shtml.
} 
(UPMC's) Center for Biosecurity held a conference to summarize important lessons learned from pandemic responses and raised policy suggestions in mitigating future infectious disease emergencies. On May 4-5th, 2010, the CDC, the National Association of County and City Health Officials (NACCHO), and other stakeholders met to review the federal, state and local policies that had an impact on local health departments' pandemic detection, response, and recovery efforts.

\subsection{An Overall Analysis of Global Prevention and Control for the Influenza Pandemic}

While modernized health care systems, antiviral drugs and vaccines represented the advantages of global response efforts this time around, factors like globalization and urbanization allowed the fastest transmission of any pandemic ever witnessed. After outbreaks occurred in multiple countries, governments worldwide immediately adopted a wide variety of proactive containment measures. While there were many successful responses, shortcomings were also exposed which incited doubt and controversy surrounding the pandemic.

\subsubsection{General Characteristics of Global Prevention and Control}

\subsubsection{Governments Played Proactive, Even Leading Roles in Response Efforts}

In regards to prevention and control measures, governments in most of the affected countries did not look lightly upon the pandemic, and they played leading roles in policy making, resource collection and allocation, as well as organization and coordination. Firstly, governments identified and allocated prevention and control organizations and accountability mechanisms at the national level. As mentioned before, some countries such as the United Kingdom and India specifically established bodies for comprehensive coordination in response to the influenza pandemic, while others like the United States - where established emergency response agencies were already in existence-launched their emergency response efforts upon the outbreak of the pandemic. The U.S. government then oversaw an organized response from varying agencies. Secondly, countries developed national-level pandemic strategies or response plans as general outlines for prevention and control efforts. Thirdly, funds for response efforts in most cases originated from the central government, where the capital was then allocated to appropriate departments based upon their responsibilities. Lastly, central governments were in charge of across-the-board organization and coordination in all aspects of the response efforts, 
especially in the provision of services, drug supplies, and vaccinations, while at the same time playing a crucial role in communication and coordination with other social service organizations, businesses, and the general public.

\subsubsection{WHO Facilitated Global Coordination and Guidance}

In the course of global responses to the sudden outbreak of the influenza pandemic, the WHO made good use of its expertise and networking strengths. With a global approach, the organization disseminated information, pushed coordination, and strengthened guidelines. It played an important role in coordinating and guiding countries' efforts to raise awareness, develop technical guidance, release pandemic information, develop vaccines, etc.

\subsubsection{Most Countries Emphasized Domestic Collaboration in Pandemic Response Efforts}

Most countries possessed an influenza prevention and control system comprised of a variety of collaborative relationships, i.e.: partnerships between central, provincial (state), and local governments, the private sector, and individuals, as well as international partnerships established through bilateral or multilateral collaboration. Each party within this system had its function and standard operating procedures, with the division of labor already institutionalized; and in implementing specific prevention and control measures, these parties were expected to fulfill their expectations and duties as stakeholders. Each stakeholder understood their role to play during the preparation, prevention, and control of the pandemic, and no major changes occurred in that respect during the pandemic. At the same time, capacity building and positioning was constantly being improved according to the different functions of each party. In addition to inter-departmental coordination and collaboration, countries like the United States also called upon the public for participation and global collaboration, which expanded collaboration as it brought in community and societal involvement.

\subsubsection{Most Countries Focused on Policy Adjustments in Prevention and Control}

During different phases of the pandemic, countries emphasized the integration of comprehensive measures and key response issues, and efforts were adjusted according to the development of the pandemic. In the early phases, prevention and control strategies were "strict," as they focused largely on containment with inspection and quarantine measures. Cases diagnosed early were treated in a timely manner to better the odds of developing a successful vaccination. At the spreading period of the pandemic, the focus shifted to clinical treatment of patients, alongside 
strengthening virus monitoring. During the post-peak period, while some countries quickly revised alert levels which reduced social impact, others had no readjustment mechanisms for policy changes in place which resulted in inefficient prevention and control.

\subsubsection{Most Countries Emphasized International and Bilateral Collaboration}

During this time, most countries recognized the importance of international collaboration. Firstly, faced with the grim situation of a pandemic gripping the globe, affected countries followed the WHO's pandemic strategies and recommendations. Combining the domestic situation with WHO's proactive policies and recommendations, most countries adopted relevant response measures. However, there were many countries that didn't adopt all of the WHO's policies and recommendation, nor did they follow all of the policy readjustments. Instead in light of their domestic situation, governments formulated their own response strategies and measures. Secondly, relatively close collaboration between countries did occur. The United States, for instance, deployed 16 personnel to Mexico including experts in influenza epidemiology, laboratory, health communications, emergency operations, information technology and veterinary sciences, who worked under PAHO, the WHO and a trilateral team of Mexican, Canadian and American experts. ${ }^{59}$ The personnel provided both technical support on the epidemiology of the virus as well as laboratory support for confirmed cases. Japan donated 100 million Japanese Yen worth of emergency materials to Mexico - the country hit the hardest by the pandemic including 190,000 masks, 3000 pairs of goggles, 3000 surgical gowns, 3000 pairs of surgical gloves, and 1370 bottles of disinfectant agents.

\subsubsection{Some Countries Focused on Risk Communication}

Strengthening risk communication has increasingly become an important part of public health emergency management. The United States' routine monitoring system provided a good foundation for risk communication. Its fast multidisciplinary information sharing, multi-level public communication (national and local), and online information management and dissemination, were crucial in coordinating programs and carrying out public health emergency management and response efforts among federal agencies and departments. The HHS continued to develop and strengthen communication as it was an important part of the public health response efforts, ${ }^{60}$ this included broad distribution of public service announcements, news reports, and other traditional media, use of social media such

\footnotetext{
${ }^{59}$ Anna Schuchat (2009b).

${ }^{60}$ See Footnote 30.
} 
as podcasts and blogs, and frequent phone conferences. The HHS issued a series of risk communication guidance documents like Communicating in a Crisis: Risk Communication Guidelines for Public Officials. Working with the DHS, the HHS also developed a National Situation Report, which was published on the DHS' website. That being said, most other countries didn't possess such robust epidemic monitoring and information collection systems, and so governments' timely explanations and communication took the forefront in response efforts. For example, India's Ministry of Health and Family Welfare appointed officials to announce latest developments to the public, disseminate public health information such as disease-related risks, personal protection measures and disease prevention guidelines via media and leaflets, and launch round-the-clock hotlines to provide citizens with pandemic guidance and facilitate the reporting of influenza cases.

\subsubsection{Controversy over WHO Response and National Prevention and Control Strategies and Measures}

\subsubsection{Controversies surrounding the appropriateness of the countries' prevention and control strategies}

Due to the many uncertainties surrounding the occurrence and development of the Influenza A (H1N1) pandemic, the level of "appropriateness" of response strategies -i.e. were they considered "lax" or "strict," "ineffective" or "overreacting" became a major controversial point surrounding the pandemic prevention and control policy.

On the one hand, based on their own pandemic situations, their preparation evaluation, and cost-benefit analyses, developed countries such as the United States, Canada, the United Kingdom, and France, adopted policies that focused more on treatment than on control. The United States, for example, in the early days of the pandemic considered Influenza A (H1N1) no bigger a threat than the seasonal influenza, so the government failed to take strict response measures, like quarantine and medical observation, which resulted in a spike in domestic infections. On October 23rd, 2009 the United States declared a national health emergency, sparking questions about the government's response efforts. While some critics questioned whether there indeed existed such an emergency, others argued that a state of emergency should have been declared from the very beginning. An article published in the New York Times in early January 2010, gave full recognition to the country's response strategy, insisting that apart from luck, the federal government's appropriate, rapid, and conservative response successfully contained the virus and minimized potential harmful effects it could've had on the economy.

On the other hand, some countries began with strict measures and relaxed them later on, causing difficulties in latent response efforts. For example, countries like Mexico declared a state of high alert immediately upon the outbreak, leading to a 
certain extent, a public panic. But after the WHO elevated the pandemic alert phase, the Mexican government rushed to lower its domestic alert level in order to ease public anxiety. Thus the public became careless, causing the increased transmission rate.

Moreover, media in Japan, France and other countries exaggerated pandemic situations that embellished "the widespread transmission" of the virus in home countries through imported cases. People became panic-stricken and it became increasingly difficult to implement proper response measures. Japan and other countries failed in resource management as they placed too much emphasis on border control and quarantine, and not enough on domestic control and detection, thus making it difficult to contain the spread of the pandemic. These actions also led to widespread criticism of government response efforts.

\subsubsection{Controversy over the WHO's Response}

Though the WHO's role in the global pandemic response efforts was widely recognized, the organization also suffered criticism as there were varied opinions about the timeliness of alert level changes and their investments in personnel and equipment. Reuters reported on April 12th, 2010, that the WHO admitted to having problems in their response efforts, including its failure to communicate the uncertainty of the new virus before it swept the globe. Some critics held that from the perspective of pandemic development, the Influenza A (H1N1) pandemic was not as dreadful as it was initially anticipated, and it was the WHO that created a global panic in its response-which caused an excess in vaccination stockpiling among some countries. Some even suspected that the IHR Emergency Committee might have had an "affair" with some drug manufacturers and was suspected of helping them seek profit by deliberately exaggerating pandemic situations so that the WHO would raise its pandemic alert to the highest level. ${ }^{61}$ In response, on April 12th, 2010, the WHO commissioned a panel of external experts to conduct an overall evaluation of the global response to the influenza pandemic in the hope of providing lessons for the future, and simultaneously to assess the global implementation of the IHR 2005. The WHO's policy evaluation comprised three main parts, i.e. capacity and preparedness, pandemic alert and risk assessment, and response. On June 10th, 2010, the WHO officially responded to and clarified such issues as to the Influenza A (H1N1) virus met the criteria for a pandemic, the severity of the pandemic, and related conflicts of interest. ${ }^{62}$

\footnotetext{
${ }^{61}$ Central People's Government of the People's Republic of China. WHO Experts Warn Global H1N1 Pandemic Still Not Over Yet. http://www.gov.cn/jrzg/2010-04/15/content_1581776.htm.

${ }^{62}$ WHO. The international response to the influenza pandemic: WHO responds to the critics. http:// www.who.int/csr/disease/swineflu/notes/briefing_20100610/en/index.html.
} 


\subsubsection{Controversy over Vaccine and Antiviral Drug Policies and Technology}

There are no international standards for vaccine allocation in mitigating the global burden of disease. While the United States began vaccinating its citizens in early October 2009 after the FDA approved on September 15th the marketing of Influenza A (H1N1) vaccines produced by CSL, MedImmune, Novartis Vaccines and Diagnostics, and Sanofi Pasteur, Mexico, which had been suffering a severer pandemic situation, was unable to launch a vaccination program until January 2010. Building a powerful global vaccine production infrastructure for influenza pandemics where countries and regions in need could acquire adequate vaccines at affordable prices became one of the hot international topics at this time.

The WHO stated that although antiviral drugs used at that time to combat influenza enjoyed complete patent protection, the organization proposed that these drugs be acquirable in the cases of public health crises. The use of antiviral drugs was hit heavily upon in the WHO's guidance documents, but, given cost issues, the use of such drugs and vaccines had little operability in most middle and low-income countries.

Moreover, some international media held that the outbreak in the United States brought to the forefront the many flaws in their healthcare system, most notably the use of old-fashioned vaccine technology and excessive reliance on vaccine manufacturers abroad. A highly controversial event also occurred during vaccination distribution: The New York City Department of Health and Mental Hygiene decided to give the small amount of vaccine available in the early phases of the pandemic to big corporations on Wall Street such as Goldman Sachs and Citibank, an act which experts believe only exacerbated public relation issues. Vaccine production and distribution became a controversial focal point during prevention and control of the pandemic as it involved multi-faceted issues such as vaccine patents, mass psychology, and social justice.

\section{References}

Anne Schuchat, M. D. (2009a). H1N1 preparedness: An overview of vaccine production and distribution, November 18, 2009. http://www.hhs.gov/asl/testify/2009/11/t20091118b.html.

Anne Schuchat, M. D. (2009b). U.S. global health response to a novel 2009-H1N1, May 6, 2009. http://www.hhs.gov/asl/testify/2009/05/t20090506b.html.

Craig Vanderwagen, W. (2009). HHS' effort to provide science-based pandemic influenza guidance for the U.S. workforce, June 16, 2009. http://www.hhs.gov/asl/testify/2009/06/ t20090616b.html.

Del Rio, C., \& Hernandez-Avila, M. (2009). Lessons from previous influenza pandemics and from the Mexican response to the current influenza pandemic. Arch Med Res, 40, 677-680.

Jesse Goodman, M. D. (2009). H1N1 preparedness: An overview of vaccine production and distribution, November 18, 2009. http://www.hhs.gov/asl/testify/2009/11/t20091118a.html.

John, T. J., \& Moorthy, M. (2010). 2009 pandemic influenza in India. Indian Pediatrics, 47(2010), $25-31$. 
Liu, G., \& Yang, L. (2009) WHO Director-General Margaret Chan says international community cannot afford to take influenza A (H1N1) pandemic lightly. Xinhuanet.com, May 19, 2009. http://news.xinhuanet.com/world/2009-05/19/content_11397804_1.htm.

Sebellus, K. (2009a). Preparing for the 2009-2010 influenza season, September 15, 2009. http:// www.hhs.gov/asl/testify/2009/09/t20090915a.html.

Sebellus, K. (2009b). 2009 H1N1 influenza: Monitoring the nation's response, October 21, 2009. http://www.hhs.gov/asl/testify/2009/10/t20091021a.html.

Weissman, D. N. (2009). Protecting the protectors: An assessment of front-line federal workers in response to the 2009-H1N1 influenza outbreak, May 14, 2009. http://www.hhs.gov/asl/testify/ 2009/05/t20090514a.html.

WHO. (2009a). Global surveillance during an influenza pandemic. Version1, updated draft April 2009. http://www.who.int/csr/resources/publications/swineflu/surveillance/en/index.html.

WHO (2009b). Assessing the severity of an influenza pandemic, May 11, 2009. http://www.who. $\mathrm{int} / \mathrm{csr} / \mathrm{disease} / \mathrm{swineflu} / \mathrm{assess} / \mathrm{disease}$ _swineflu_assess_20090511/en/index.html.

Willam Corr, J. D. (2009). 2009-H1N1 influenza: HHS preparedness and response efforts, July 29, 2009. http://www.hhs.gov/asl/testify/2009/07/t20090729b.html.

Xiaoming, R. (2009). Characteristics of the India's public health emergency management system: From a perspective of influenza A (N1H1) preparedness and response. Global Science, Technology and Economy Outlook, Issue 7.

Zhang, Z. (2009) Influenza A pandemic moves into a new phase, WHO changes way of epidemic reporting. Xinhuanet.com, July 17, 2009. http://news.xinhuanet.com/newscenter/2009-07/17/ content_11723758.htm. 